Supporting Information

\title{
Molecular Simulation on Thermodynamic Swelling Behavior of Bilayer Graphene Oxide in Solvents
}

Wei Tong, Dandan Cui, Zhijun Xu, and Xiaoning Yang*

State Key Laboratory of Material Chemical Engineering,

College of Chemical Engineering,

Nanjing Tech University,

Nanjing, 211816, China. 
(a)

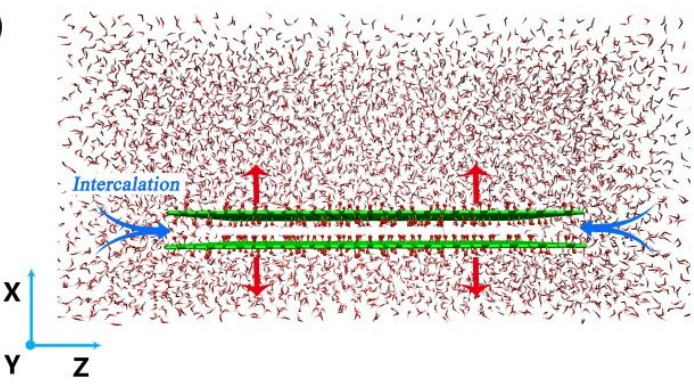

(b)

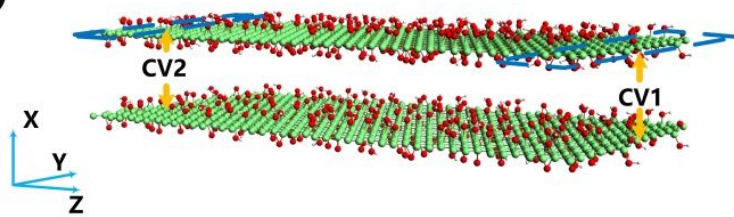

(c)
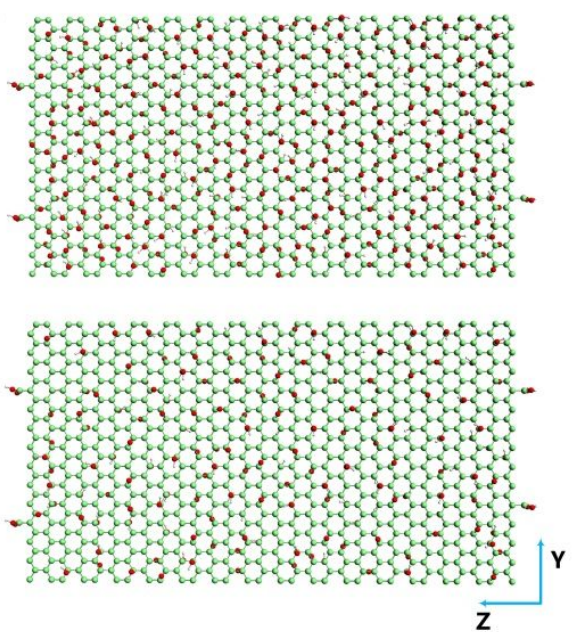

Figure S1. (a) The schematic of simulation system with bilayer GO immersed in solvent, (b) the schematic definitions of two collective variables (CV1 and CV2) for the simulation, and (c) surface structures of selected GO sheets. 


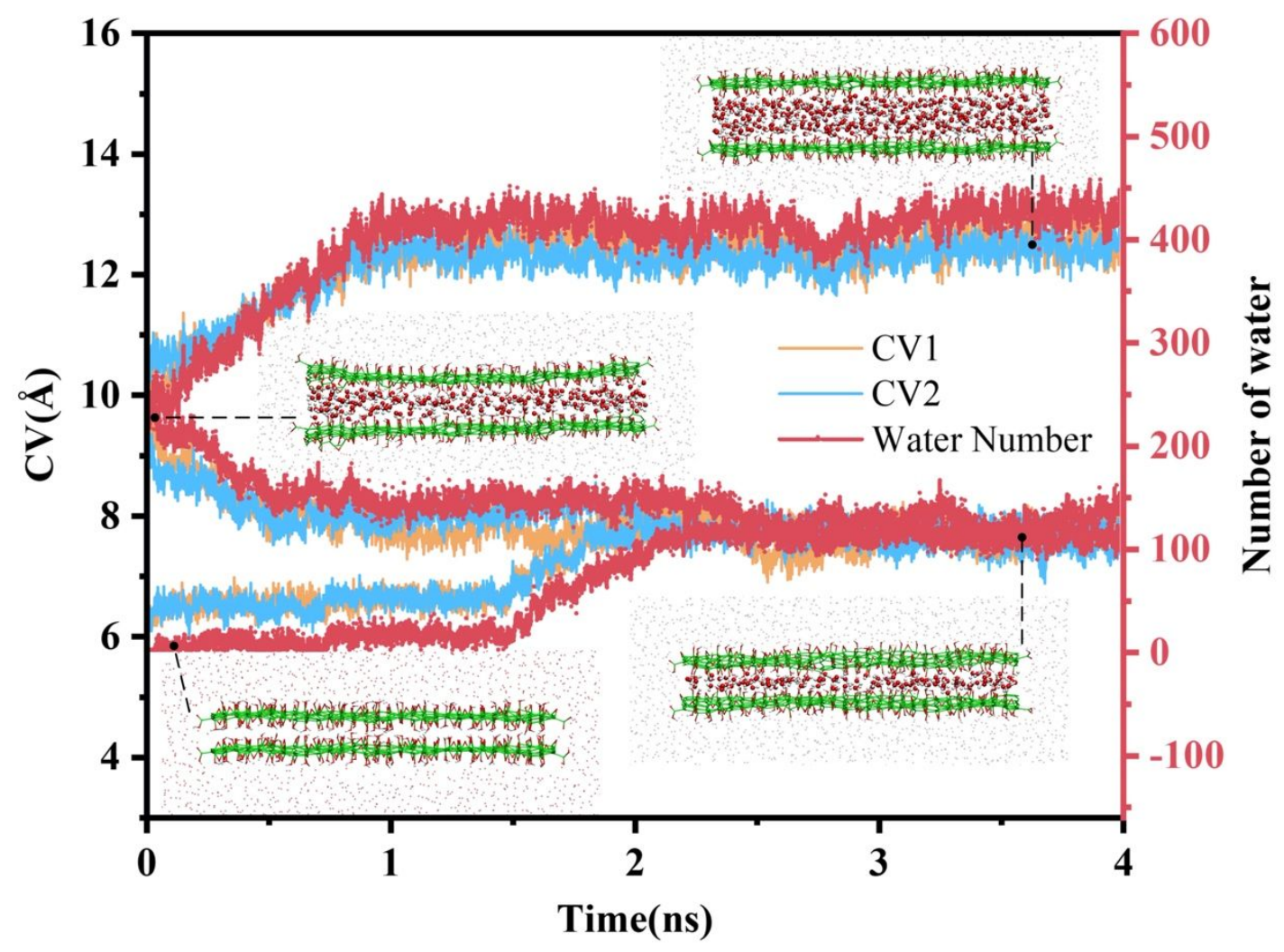

Figure S2. Evolution of CV1/CV2 and interlayer water number with simulation time under different initial interlayer spacings for the GO1 system. With the initial CV1 and CV2 of $6 \AA$, the final separation distance can reach around $7.8 \AA$ along with the water insertion, corresponding to the first minimum free-energy point. This means that the bilayer GO could spontaneously have a slight lattice expansion and the water intercalation could attenuate the attractive interaction between GO sheets. However, when the GO sheets have the initial CV1 and CV2 of $10.2 \AA$, two completely different contacting performances can be observed: one is toward a larger separation distance $(\sim 12.4 \AA)$ with the interlayer water insertion; and another is back to a smaller separation of $7.8 \AA$ with the interlayer water exclusion. The dynamic MD simulation shows consistent swelling behavior with the free-energy profile from MTD simulation. 


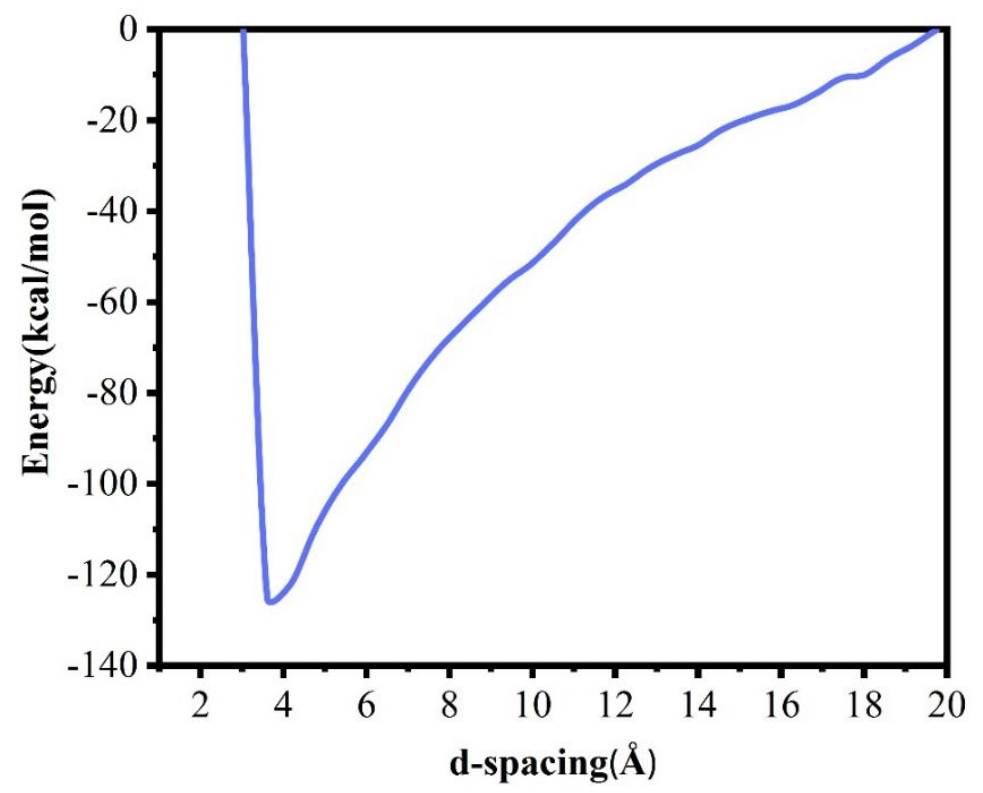

Figure S3. The 1-D free-energy profile of the hydration swelling bilayer graphene in water. 


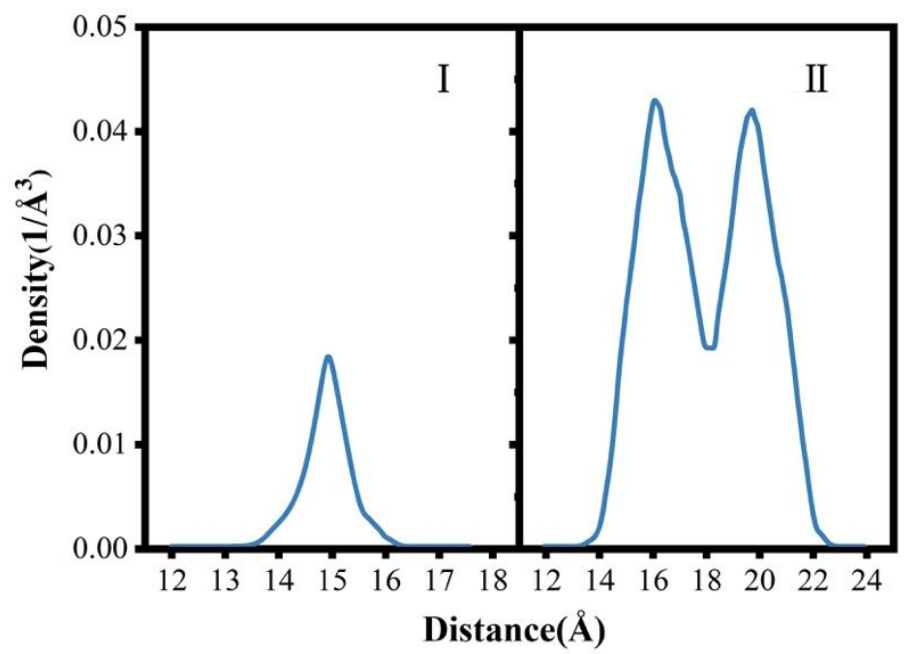

Figure S4. Density distributions of confined water oxygen atoms within the interlayer spaces at critical positions of GO1-5wt $\%$. 


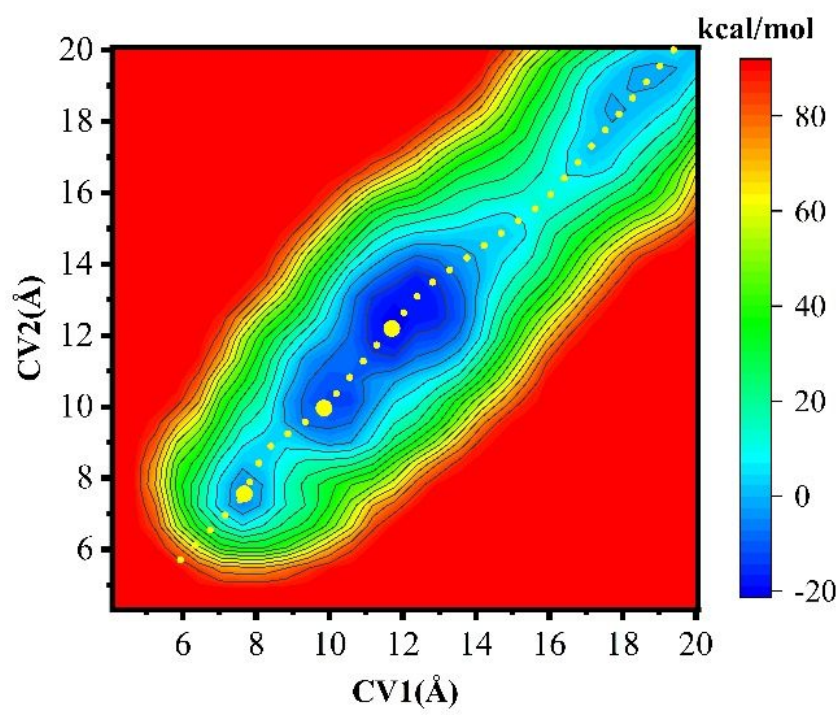

Figure S5. The 2-D free-energy surface of the swelling process for the GO1-15wt \% system. 

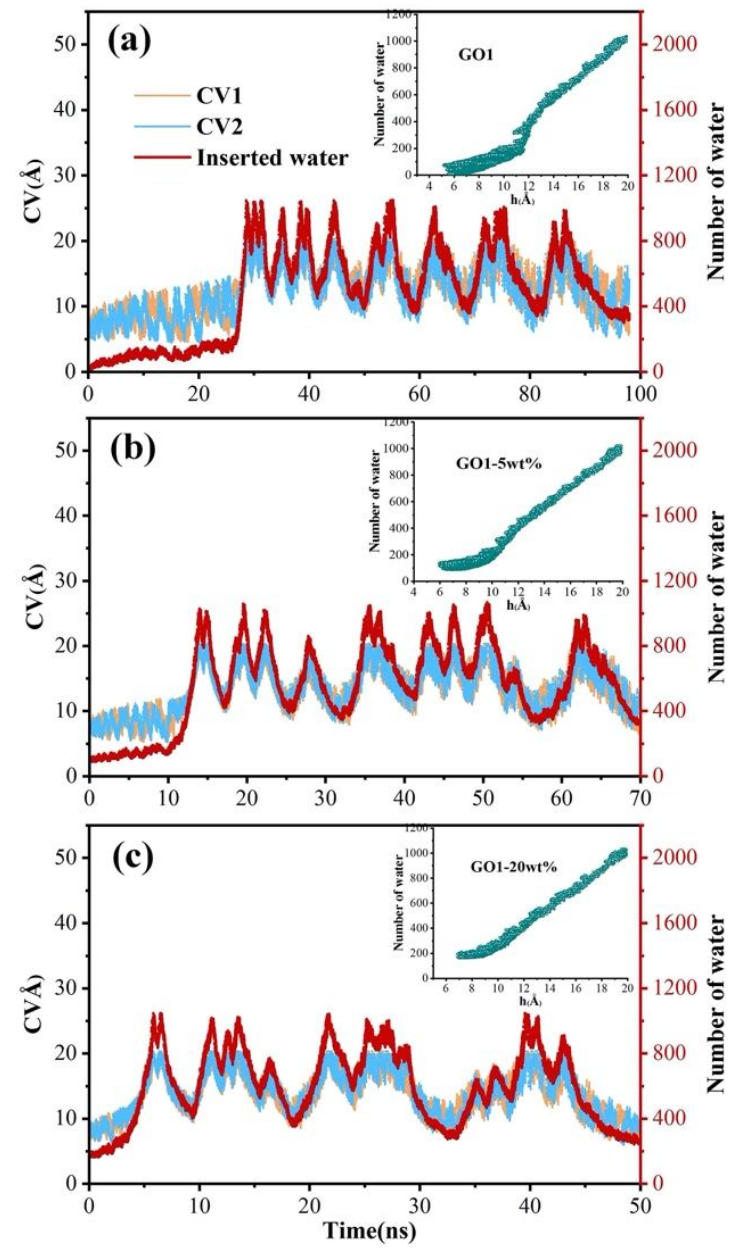

Figure S6. CVs (CV1:blue,CV2:orange) sampling and the variation of inserted water molecules with the simulation times in the hydration swelling MTD simulation for three systems, GO1(a),GO1-5wt\%(b), GO1-20wt\%(c). Insets show the variation of average $\mathrm{d}$-spacing (h) with the inserted water molecules. 

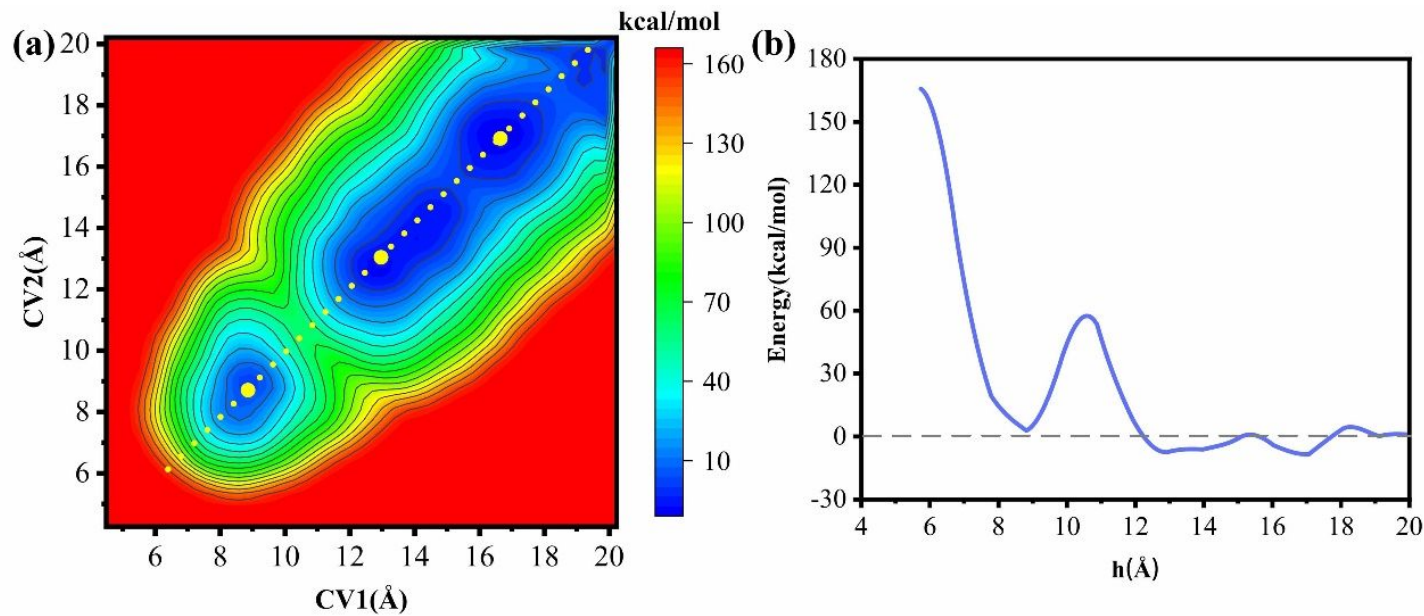

Figure S7. (a) The 2-D free-energy surface of the swelling process for the GO1 system with a small amount ( $5 \mathrm{wt} \%$ ) of initial water in pure ethanol; (b) The corresponding 1-D free-energy profile along the yellow dashed line in the free-energy surface. 


\section{HB intermittent time-correlation fucntion}

The intermittent time-correlation function $C_{H B}(t)$ of the HB between the surface oxygen-containing functional groups and the interlayer water molecules can be applied to describe the HB stability. The time-correlation function is calculated:

$$
C_{H B}(t)=\frac{\langle h(0) H(t)\rangle}{\langle h(0) h(0)\rangle}
$$

where $C_{H B}(t)=1$ means that there are hydrogen bonds between water molecules and the functional groups on the GO surface from 0 to $t$ time. If the hydrogen bonds disappear, then $C_{H B}(t)=0$. We analyzed the forming $\mathrm{H}$-bonds ${ }^{1}$ between the GO sheets and the confined water molecules, in which a geometric $\mathrm{H}$-bonding criterion was employed. ${ }^{2}$

\section{References}

1. Medhekar, N. V.; Ramasubramaniam, A.; Ruoff, R. S.; Shenoy, V. B., Hydrogen Bond Networks in Graphene Oxide Composite Paper: Structure and Mechanical Properties. Acs Nano 2010, 4, 2300-2306.

2. Luzar, A.; Chandler, D., Hydrogen-bond kinetics in liquid water. Nature 1996, 379, $55-57$. 